\title{
Testing the quantum superposition principle in the frequency domain
}

\author{
M. Bahrami, ${ }^{1, *}$ A. Bassi, ${ }^{1,2, \dagger}$ and H. Ulbricht ${ }^{3, \dagger}$ \\ ${ }^{1}$ Department of Physics, University of Trieste, Strada Costiera 11, 34014 Trieste, Italy \\ ${ }^{2}$ Istituto Nazionale di Fisica Nucleare, Trieste Section, Via Valerio 2, 34127 Trieste, Italy \\ ${ }^{3}$ School of Physics and Astronomy, University of Southampton, Southampton SO17 1BJ, United Kingdom \\ (Received 23 September 2013; revised manuscript received 22 November 2013; published 28 March 2014)
}

\begin{abstract}
We study how photon emission of a two-level system is modified if the superposition principle is violated. We solve the relevant equations of motion. We quantify the magnitude of the new spectral effects for relevant collapse models to illustrate our theoretical results. We show how these effects can be distinguished from those of standard environmental decoherence. We apply our result to physically interesting systems and suggest that accurate-enough spectroscopic experiments are within reach with current technology.
\end{abstract}

DOI: 10.1103/PhysRevA.89.032127

PACS number(s): 03.65.Ta, 42.50.Xa, 42.62.Fi

\section{INTRODUCTION}

Quantum theory is an extremely successful theory of microscopic phenomena. To this day macroscopic systems have always been found to behave according to classical laws and the open question is if and how they can show quantum behavior. One possibility to address this quantum-classical divide is to investigate whether the quantum superposition principle is violated for macroscopic systems that explain the observed classical behavior. Is the loss of quantum superposition of macroscopic systems due to a universal mechanism, which affects all objects in a given parameter range and which cannot be avoided, or is it because of environmental interactions as explained by decoherence theory? In the latter case, the control of the environment can be used to tune the decoherence effect, which is believed to be impossible for a universal mechanism.

To shine light on this question, experiments are needed to probe the regime of this quantum-to-classical transition. The main reason why quantum properties of macroscopic objects are so difficult to observe is that they are highly unstable against external perturbations. Decoherence theory explains well how local interactions with the environment can rapidly destroy quantum coherence before it can even be observed [1,2]. Detailed experimental studies have been performed to investigate dominating decoherence effects for molecules in spatial superposition states, such as collisional decoherence [3] and the emission of blackbody photons [4]. While this has been proven by an experiment, we cannot be sure if that already provides the final answer to our question, which means that more experiments with even more macroscopic quantum systems are required. The system parameters to quantify if a system is sufficiently macroscopic are given by the mass of the system, the spatial size of the superposition state, and the lifetime of the superposition, which have been recently united to the so-called macroscopicity [5]. The actual macroscopicity record is more than $10^{4}$ amu (atomic mass units) for a spatial size of the superposition of about $100 \mathrm{~nm}$ and a lifetime of the superposition of some $10 \mathrm{~ms}$, measured by molecule interferometry [6].

\footnotetext{
*Mohammad.Bahrami@ts.infn.it

†bassi@ts.infn.it

${ }^{\ddagger}$ h.ulbricht@soton.ac.uk
}

Along with the decoherence prediction where local interactions with the environment play the key role for the appearance of the classical behavior of physical systems, there have been speculations that the superposition principle might be violated at the macroscopic level in a universal fashion [7-10], either by interactions with the gravitational field as in the Diósi-Penrose (DP) model [11] and the Schrödinger-Newton equation [12], or by intrinsic nonlinear effects built in the dynamics as in the Ghirardi-Rimini-Weber (GRW) and continuous spontaneous localization (CSL) models [13-15]. That provides an alternative to explain the quantum-to-classical transition for macroscopic systems, and therefore, as much as decoherence theory, it might provide insight to a new underlying theory of nature [15]. The aforementioned matter-wave experiments will need another two orders of magnitude in mass to test the CSL according to Adler [16].

In the past 10 or so years, there has been rapid experimental progress in revealing quantum superpositions for large particles like organic molecules in matter-wave interferometers with tiny de Broglie wavelengths of down to few hundred femtometers [17]. Such objects were successfully decoupled from environmental noises, thus overcoming the technological limit and thereby extending the realm of quantum theory to new regimes [6,17-24]. Technological challenges to extend those experiments to even larger particles are summarized in detail elsewhere [25]. In brief, the main challenge is to prepare a spatial superposition state of free-space single particles of increasing mass, which requires the generation of spatially coherent beams of particles, which need to be detectable with sufficient efficiency. This requires the further development of molecule and cluster beam techniques. While the aforementioned molecule interference experiments are going on, quite a few new experimental schemes to test the superposition principle have been proposed [26-29]. One technical bottleneck for all of those experiments is the cooling of certain degrees of freedom of particles to low temperatures, a technology which is just emerging right now [30,31]. To push the macroscopicity even further, Earth's gravitational field has to be compensated for [32-34] or avoided by performing the experiment in space [35]. All this sums up to immense experimental challenges to generate a macroscopic quantum superposition state and we are therefore, in this paper, interested to propose a way to test the quantum superposition principle. 


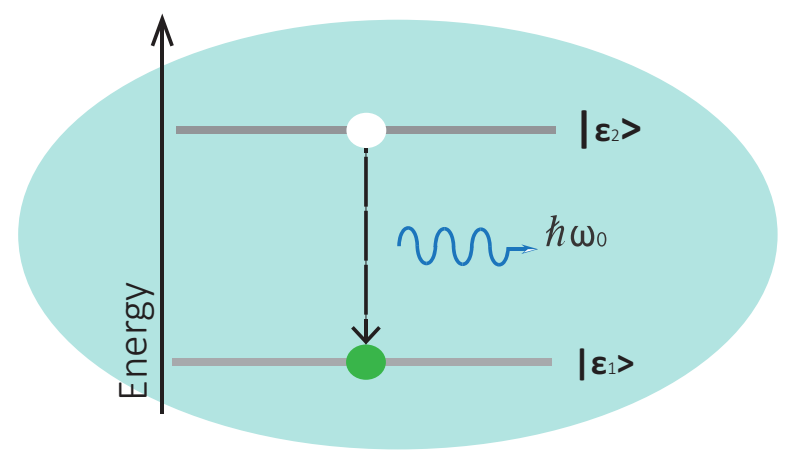

(a)

(b)

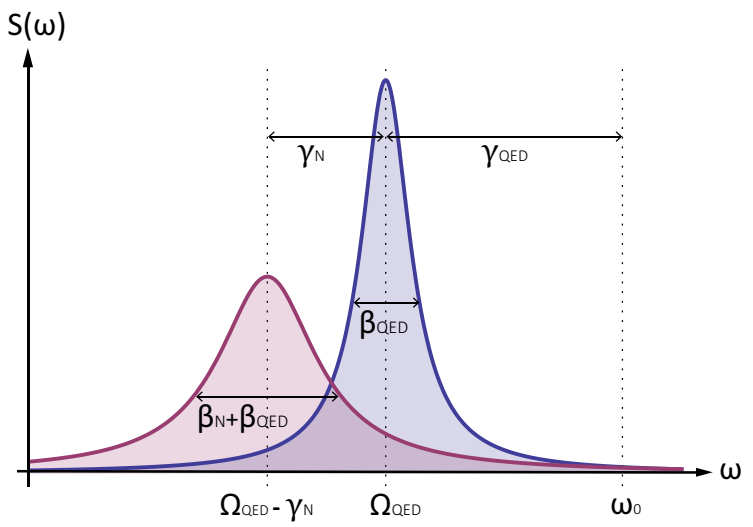

FIG. 1. (Color online) Violations of the quantum superposition principle predict a broadening and a shift of line shapes of light emitted by radiative transitions among energy levels, in addition to those generated by vacuum fluctuations and interactions with external environments (decoherence). Panel (a) shows the model system which we discuss. It is the energy diagram of the two-level system emitting photons at optical frequency $\omega_{0}$ for the dipole-allowed transition from excited state $\left|\varepsilon_{2}\right\rangle$ to a lower state $\left|\varepsilon_{1}\right\rangle$. (b) Spectral density plotted vs frequency. The blue Lorentzian shows the emission spectral line according to standard QED, where $\gamma_{\mathrm{QED}}$ is the Lamb shift with respect to $\omega_{0}$ and spectral width is given by QED broadening $\beta_{\mathrm{QED}}$. The red curve illustrates the additional broadening $\left(\beta_{\mathrm{N}}\right)$ and shift $\left(\gamma_{\mathrm{N}}\right)$ if the quantum superposition principle is violated.

By assuming a universal (omnipresent and not environmental) mechanism to be responsible for the quantum-to-classical transition of macroscopic systems, we work out how it affects the linewidth and frequency of photon emission lines. The effect of such a universal mechanism is a perturbation of any two-level system, which results in the loss of coherence of the emitted light. For the spectral density of emitted radiation from a generic two-level system (see Fig. 1), we obtain

$$
\mathcal{S}(\omega)=\frac{1}{\pi}\left[\frac{\beta}{\beta^{2}+(\omega-\Omega)^{2}}\right],
$$

which is a Lorentzian distribution with the central frequency $\Omega \simeq \omega_{0}-\gamma_{\mathrm{QED}}-\gamma_{\mathrm{N}}$, where $\gamma_{\mathrm{QED}}$ is the shift induced by vacuum fluctuations (Lamb shift) and $\gamma_{\mathrm{N}}$ is the shift induced by the violation of quantum linearity; and the full width at half maximum of $2 \beta=2 \beta_{\mathrm{QED}}+2 \beta_{\mathrm{N}}$, where $\beta_{\mathrm{QED}}$ is the broadening induced by vacuum fluctuations (natural broadening) and $\beta_{\mathrm{N}}$ is the broadening induced by nonlinear effects. For $\beta_{\mathrm{N}}=\gamma_{\mathrm{N}}=0$, the standard quantum optical spectrum is recovered.

It is important to note that the nonlinear effect scales with mass of the system: It is tiny and out of reach for experiments for a small system, like the hydrogen atom, but prominent and possible to be observed for larger systems, such as macromolecules. The macroscopicity measure from above applies in the same way to our spectroscopic scheme. However, the experimental challenge for observation might be smaller; indeed it is of completely different nature compared to matterwave interferometry experiments as no spatial superposition state has to be actively generated.

The outline of the paper is the following. In Sec. II we present the mathematical setup for analyzing the problem. In Sec. III we solve the equations of motion and derive Eq. (1). In Sec. IV we apply the results to the specific cases of the CSL and DP collapse models. In Sec. V we estimate the Lamb shift and broadening effects for relevant physical systems: the hydrogenlike system, the harmonic oscillator, and the doublewell potential. In Sec. VI we estimate decoherence effects, and we show how collapselike effects can be distinguished from standard decoherence effects. Conclusions follow. We believe that this work holds as motivation to push present limits in high-resolution spectroscopy.

\section{MATHEMATICAL SETUP}

It was first proven by Gisin [36] that, in order to avoid superluminal signaling, nonlinear terms can be added to the Schrödinger equation only combined with stochastic terms in such a way that the equivalence relation among statistical ensembles of states is preserved by the dynamics [36-38]. In more mathematical terms, this means that the modified dynamics for the wave function must generate a closed linear dynamics for the density matrix. Therefore, we start from a dynamics for the density matrix of the form [39]

$$
\begin{aligned}
\frac{d \hat{\rho}_{t}}{d t}= & -\frac{i}{\hbar}\left[\hat{H}_{0}, \hat{\rho}_{t}\right]+\lambda \sum_{k=1}^{n}\left(\hat{L}_{k} \hat{\rho}_{t} \hat{L}_{k}^{\dagger}\right. \\
& \left.-\frac{1}{2} \hat{L}_{k}^{\dagger} \hat{L}_{k} \hat{\rho}_{t}-\frac{1}{2} \hat{\rho}_{t} \hat{L}_{k}^{\dagger} \hat{L}_{k}\right),
\end{aligned}
$$

where the Lindblad operators $\hat{L}_{k}$ can describe decoherence effect or, as is the case here, intrinsic nonlinearities in the dynamics for the wave function. Collapse models induce a dynamics of this type, but here we want to stay more general. The most convenient unraveling of Eq. (2) for solving the equations of motions, is given in terms of a stochastic potential added to the Schrödinger equation [40-44],

$$
i \hbar \frac{d}{d t} \psi_{t}=\left[\hat{H}_{0}+\hat{V}_{t}\right] \psi_{t}, \quad \hat{V}_{t}=-\hbar \sqrt{\lambda} \sum_{k=1}^{n} \hat{L}_{k} \xi_{t}^{(k)}
$$


where $\xi_{t}^{(k)}$ are $n$ independent white noises. Here, we have assumed that the Lindblad operators $\hat{L}_{k}$ are self-adjoint, which is the case for most proposals for nonlinear and stochastic modifications of the Schrödinger equation.

Since by violations of the superposition principle we mean superpositions in space, the Lindblad operators are taken as functions of space variables; therefore, we have

$$
\hat{V}_{t}=-\hbar \sqrt{\lambda} \int d^{3} x \hat{L}(\mathbf{x}) \xi_{t}(\mathbf{x}),
$$

where $\xi_{t}(\mathbf{x})$ is a noise field, white in both space and time. Note that, in this form, the dynamical equation is still linear. As discussed several times in the literature [40-44], the effects of nonlinear terms introduced in the Schrödinger equation, at the statistical level, can be mimicked also by linear random potentials. For individual realizations of the noise, the affects are very different (those of a linear dynamics vs those of a nonlinear one), while at the statistical level they coincide, if the potential is suitably chosen.

\section{A. Two-level systems}

We consider the situation in which the system's dynamics effectively involves only two levels, whose transition dipole matrix element is not zero $\left(\mathbf{d}_{12}=\left\langle\varepsilon_{1}|\hat{\mathbf{d}}| \varepsilon_{2}\right\rangle \neq 0\right.$, with the dipole operator defined as $\left.\hat{\mathbf{d}}=\sum_{i} e_{i} \hat{\mathbf{q}}_{i}\right)$. This means that the higher energy level eventually decays to the lower one, by emitting a photon. Therefore, the standard quantum Hamiltonian characterizing the interaction between a two-level system and a quantized radiation field can be, in the dipole approximation, written in the form [45-48]

$$
\begin{aligned}
\hat{H}_{0}= & \hbar \sum_{s, \mathbf{k}} \omega \hat{a}_{s, \mathbf{k}}^{\dagger} \hat{a}_{s, \mathbf{k}}+\left(\omega_{0} / 2\right) \hat{\sigma}_{z} \\
& -i \omega_{0} \hbar \sum_{s, \mathbf{k}}\left[g_{s, \mathbf{k}}\left(\hat{\sigma}_{+}-\hat{\sigma}_{-}\right) \hat{a}_{s, \mathbf{k}}-\text { H.c. }\right],
\end{aligned}
$$

with $g_{s, \mathbf{k}}=\left(2 \epsilon_{0} \hbar \omega L^{3}\right)^{-1 / 2} \mathbf{d}_{12} \cdot \mathbf{e}_{s, \mathbf{k}}$ the coupling constant of radiation matter, $\hat{\sigma}_{+}=\left|\varepsilon_{2}\right\rangle\left\langle\varepsilon_{1}\left|, \hat{\sigma}_{-}=\right| \varepsilon_{1}\right\rangle\left\langle\varepsilon_{2}\right|, \hat{\sigma}_{z}=\hat{\sigma}_{+} \hat{\sigma}_{-}-$ $\hat{\sigma}_{-} \hat{\sigma}_{+}, \omega_{0}=\left(\varepsilon_{2}-\varepsilon_{1}\right) / \hbar$, and $\left\{\left|\varepsilon_{1}\right\rangle,\left|\varepsilon_{2}\right\rangle\right\}$ the two levels of matter. All other terms have the usual meaning.

The two-level representation of $\hat{V}_{t}$ is obtained by calculating $\left\langle\varepsilon_{\alpha}\left|\hat{V}_{t}\right| \varepsilon_{\beta}\right\rangle$ with $\alpha, \beta=1,2$. In general, one has

$$
\hat{V}_{t}=-\hbar\left(\sqrt{\lambda_{z}} w_{t}^{(z)} \hat{\sigma}_{z}+\sqrt{\lambda_{x}} w_{t}^{(x)} \hat{\sigma}_{x}+\sqrt{\lambda_{x}} w_{t}^{(y)} \hat{\sigma}_{y}\right),
$$

where $\lambda_{i}(i=x, y, z)$ are collapse rates and $w_{t}^{(i)}$ are three white noises. Eigenenergies are real functions in most cases; thus, one finds $\lambda_{y}=0$. Therefore, $\hat{V}_{t}$ simplifies to

$$
\hat{V}_{t}=-\hbar\left(\sqrt{\lambda_{z}} w_{t}^{(z)} \hat{\sigma}_{z}+\sqrt{\lambda_{x}} w_{t}^{(x)} \hat{\sigma}_{x}\right) .
$$

This is the starting point of our calculation.

\section{SOLUTION OF THE EQUATIONS OF MOTION: SHIFT AND BROADENING}

The radiative corrections of the nonlinearities appear in a very natural way from the formulation of the spectral density of emitted light [45-48], which is given by the stochastic expectation (averaging) of the Fourier transform of the normalized dipole-dipole autocorrelation function. The dipole-dipole autocorrelation function is given by $\left\langle\hat{\sigma}_{+}(t+\tau) \hat{\sigma}_{-}(t)\right\rangle$.

Given the Hamiltonian $\hat{H}=\hat{H}_{0}+\hat{V}_{t}$, the Heisenberg equations of motion for the Pauli operators are

$$
\begin{aligned}
\frac{d}{d t} \hat{\sigma}_{z}(t)= & -2 \beta_{\mathrm{QED}}\left[\hat{\sigma}_{z}(t)+\hat{I}\right]-2 \omega_{0}\left[\hat{\sigma}_{x}(t) \mathbf{d}_{12} \cdot \hat{\mathbf{A}}_{\mathrm{free}}^{(+)}(0, t)\right. \\
& \left.+\mathbf{d}_{12} \cdot \hat{\mathbf{A}}_{\mathrm{free}}^{(-)}(0, t) \hat{\sigma}_{x}(t)\right]-2 \sqrt{\lambda_{x}} w_{t}^{(x)} \hat{\sigma}_{y}(t) \\
\frac{d}{d t} \hat{\sigma}_{y}(t)= & -\left(\Omega_{\mathrm{QED}}-2 \sqrt{\lambda_{z}} w_{t}^{(z)}\right) \hat{\sigma}_{x}(t) \\
& -\beta_{\mathrm{QED}} \hat{\sigma}_{y}(t)+2 \sqrt{\lambda_{x}} w_{t}^{(x)} \hat{\sigma}_{z}(t) \\
\frac{d}{d t} \hat{\sigma}_{x}(t)= & -\left(\Omega_{\mathrm{QED}}-2 \sqrt{\lambda_{z}} w_{t}^{(z)}\right) \hat{\sigma}_{y}(t)-\beta_{\mathrm{QED}} \hat{\sigma}_{x}(t) \\
& +2 \omega_{0}\left[\hat{\sigma}_{z}(t) \mathbf{d}_{12} \cdot \hat{\mathbf{A}}_{\mathrm{free}}^{(+)}(0, t)\right. \\
& \left.+\mathbf{d}_{12} \cdot \hat{\mathbf{A}}_{\mathrm{free}}^{(-)}(0, t) \hat{\sigma}_{z}(t)\right]
\end{aligned}
$$

with $\Omega_{\mathrm{QED}}=\omega_{0}-\gamma_{\mathrm{QED}}$, where

$$
\gamma_{\mathrm{QED}}=\frac{\omega_{0}^{2}\left|\mathbf{d}_{12}\right|^{2}}{3 \epsilon_{0} \hbar \pi^{2} c^{3}}\left(\mathcal{P} \int_{0}^{\infty} \frac{d \omega \omega}{\omega-\omega_{0}}-\mathcal{P} \int_{0}^{\infty} \frac{d \omega \omega}{\omega+\omega_{0}}\right)
$$

is the Lamb shift, where $\mathcal{P}$ is the Cauchy principal part, which can be normalized in the standard fashion [49]; the parameter

$$
\beta_{\mathrm{QED}}=\frac{\omega_{0}^{3}\left|\mathbf{d}_{12}\right|^{2}}{6 \pi \epsilon_{0} \hbar c^{3}}
$$

is the standard spontaneous emission rate; and

$$
\mathbf{d}_{12} \cdot \hat{\mathbf{A}}_{\text {free }}^{(+)}(0, t)=\sum_{s, \mathbf{k}} g_{s, \mathbf{k}} e^{-i \omega t} \hat{a}_{s, \mathbf{k}}(0) .
$$

We now average Eqs. (8), (9), and (10) over the initial state $|\psi\rangle|0\rangle$, according to which matter is in a generic state $|\psi\rangle$ and the radiation field in the vacuum state. Therefore, we get

$$
\begin{aligned}
\frac{d}{d t}\left\langle\hat{\sigma}_{z}(t)\right\rangle=-2 & \beta_{\mathrm{QED}}\left(\left\langle\hat{\sigma}_{z}(t)\right\rangle+1\right)-2 \sqrt{\lambda_{x}} w_{t}^{(x)}\left\langle\hat{\sigma}_{y}(t)\right\rangle, \\
\frac{d}{d t}\left\langle\hat{\sigma}_{y}(t)\right\rangle= & \left(\Omega_{\mathrm{QED}}-2 \sqrt{\lambda_{z}} w_{t}^{(z)}\right)\left\langle\hat{\sigma}_{x}(t)\right\rangle-\beta_{\mathrm{QED}}\left\langle\hat{\sigma}_{y}(t)\right\rangle \\
& +2 \sqrt{\lambda_{x}} w_{t}^{(x)}\left\langle\hat{\sigma}_{z}(t)\right\rangle, \\
\frac{d}{d t}\left\langle\hat{\sigma}_{x}(t)\right\rangle=- & \left(\Omega_{\mathrm{QED}}-2 \sqrt{\lambda_{z}} w_{t}^{(z)}\right)\left\langle\hat{\sigma}_{y}(t)\right\rangle-\beta_{\mathrm{QED}}\left\langle\hat{\sigma}_{x}(t)\right\rangle .
\end{aligned}
$$

The above stochastic differential equations should be understood in the Stratonovich sense. Since we want to compute stochastic averages, it is more convenient to switch to the Itô formalism. To this end, one can use Eqs. (10.2.5) to (10.2.7) of Ref. [50]. Then, once expressed in the Itô form, by using theorem (8.5.5) of Ref. [50], one can prove that the stochastic 
expectations satisfy the equations

$$
\begin{aligned}
\frac{d}{d t} \mathbb{E}\left(\left\langle\hat{\sigma}_{z}(t)\right\rangle\right)= & -2\left(\beta_{\mathrm{QED}}+\lambda_{x}\right) \mathbb{E}\left(\left\langle\hat{\sigma}_{z}(t)\right\rangle\right)-2 \beta_{\mathrm{QED}} \\
\frac{d}{d t} \mathbb{E}\left(\left\langle\hat{\sigma}_{y}(t)\right\rangle\right)= & \Omega_{\mathrm{QED}} \mathbb{E}\left(\left\langle\hat{\sigma}_{x}(t)\right\rangle\right) \\
& -\left(\beta_{\mathrm{QED}}+2 \lambda_{x}+2 \lambda_{z}\right) \mathbb{E}\left(\left\langle\hat{\sigma}_{y}(t)\right\rangle\right) \\
\frac{d}{d t} \mathbb{E}\left(\left\langle\hat{\sigma}_{x}(t)\right\rangle\right)= & -\Omega_{\mathrm{QED}} \mathbb{E}\left(\left\langle\hat{\sigma}_{y}(t)\right\rangle\right)-\left(\beta_{\mathrm{QED}}+2 \lambda_{z}\right) \mathbb{E}\left(\left\langle\hat{\sigma}_{x}(t)\right\rangle\right)
\end{aligned}
$$

whose solutions give

$$
\begin{aligned}
\mathbb{E}\left(\left\langle\hat{\sigma}_{z}(t)\right\rangle\right)= & {\left[\frac{\beta_{\mathrm{QED}}}{\beta_{\mathrm{QED}}+\lambda_{x}}+\left\langle\hat{\sigma}_{z}(0)\right\rangle\right] } \\
& \times e^{-2\left(\beta_{\mathrm{QED}}+\lambda_{x}\right) t}-\frac{\beta_{\mathrm{QED}}}{\beta_{\mathrm{QED}}+\lambda_{x}}, \\
\mathbb{E}\left(\left\langle\hat{\sigma}_{+}(t) \hat{\sigma}_{-}(t)\right\rangle\right)= & \frac{1}{2}\left\{\left[\frac{\beta_{\mathrm{QED}}}{\beta_{\mathrm{QED}}+\lambda_{x}}+\left\langle\hat{\sigma}_{z}(0)\right\rangle\right]\right. \\
& \left.\times e^{-2\left(\beta_{\mathrm{QED}}+\lambda_{x}\right) t}+\frac{\lambda_{x}}{\beta_{\mathrm{QED}}+\lambda_{x}}\right\} .
\end{aligned}
$$

Here $\hbar \omega_{0} \mathbb{E}\left(\left\langle\hat{\sigma}_{z}(t)\right\rangle\right)$ gives the rate of energy emission by matter; and $\mathbb{E}\left(\left\langle\hat{\sigma}_{+}(t) \hat{\sigma}_{-}(t)\right\rangle\right)$ represents the change in the population of the excited state $\left|\varepsilon_{2}\right\rangle$. When the initial state is $\left|\varepsilon_{1}\right\rangle$, we have $\left\langle\hat{\sigma}_{z}(0)\right\rangle=-1$, and for $\left|\varepsilon_{2}\right\rangle$ we have $\left\langle\hat{\sigma}_{z}(0)\right\rangle=1$.
On the other hand, for both initial states $\left|\varepsilon_{1,2}\right\rangle|0\rangle$ we get $\mathbb{E}\left(\left\langle\hat{\sigma}_{-}(t)\right\rangle\right)=\mathbb{E}\left(\left\langle\hat{\sigma}_{+}(t)\right\rangle\right)=0$.

Using Eq. (18), one can compute, for example, the mean light intensity of emitted radiation in the far-field limit [45-48] as

$$
\begin{aligned}
\langle\hat{I}(\mathbf{r}, t)\rangle= & \left(\frac{\omega_{0}^{2}\left|\mathbf{d}_{12}\right|}{8 \pi \varepsilon_{0} c^{2} r}\right)^{2}\left(1-\frac{1}{2} \sin ^{2} \theta\right) \\
& \times\left\{\left[\frac{\beta_{\mathrm{QED}}}{\beta_{\mathrm{QED}}+\lambda_{x}}+\left\langle\hat{\sigma}_{z}(0)\right\rangle\right] e^{-2\left(\beta_{\mathrm{QED}}+\lambda_{x}\right)\left(t-\frac{r}{c}\right)}\right. \\
& \left.+\frac{\lambda_{x}}{\beta_{\mathrm{QED}}+\lambda_{x}}\right\} ; \quad t>\frac{r}{c}
\end{aligned}
$$

with $\theta$ the polar angle of the $\mathbf{r}$ vector, and the complex dipole moment $\mathbf{d}_{12}$ lies in the $x y$ plane, where $\mathbf{r}$ is the vector connecting the center-of-mass of system to the detector. $\langle\hat{I}(\mathbf{r}, t)\rangle$ is a very interesting quantity for experimental research; however, here we are concerned with the spectral density of emitted light, which we now compute.

The exponential nature of energy decay, as given by Eqs. (17) and (19), suggests that the spectral distribution of the emitted radiation is Lorentzian. The explicit mathematical form of spectral density can be obtained by computing the dipole-dipole autocorrelation function, $\mathbb{E}\left(\left\langle\hat{\sigma}_{+}(t+\tau) \hat{\sigma}_{-}(t)\right\rangle\right)$, and then using the Wiener-Khinchin theorem [45]. The time derivative of this autocorrelation function can be obtained by making the change $t \rightarrow t+\tau$ in Eqs. (8), (9), and (10) and then writing down the derivatives with respect to $\tau$. After multiplying the result from the right by $\hat{\sigma}_{-}(t)$ and then taking the quantum average over the initial state $|\psi\rangle|0\rangle$, we find

$$
\begin{gathered}
\frac{d}{d \tau}\left\langle\hat{\sigma}_{z}(t+\tau) \hat{\sigma}_{-}(t)\right\rangle=-2 \beta_{\mathrm{QED}}\left(\left\langle\hat{\sigma}_{z}(t+\tau) \hat{\sigma}_{-}(t)\right\rangle+\left\langle\hat{\sigma}_{-}(t)\right\rangle\right)-2 \sqrt{\lambda_{x}} w_{\tau}^{(x)}\left\langle\hat{\sigma}_{y}(t+\tau) \hat{\sigma}_{-}(t)\right\rangle \\
\frac{d}{d \tau}\left\langle\hat{\sigma}_{y}(t+\tau) \hat{\sigma}_{-}(t)\right\rangle=\left(\Omega_{\mathrm{QED}}-2 \sqrt{\lambda_{z}} w_{\tau}^{(z)}\right)\left\langle\hat{\sigma}_{x}(t+\tau) \hat{\sigma}_{-}(t)\right\rangle-\beta_{\mathrm{QED}}\left\langle\hat{\sigma}_{y}(t+\tau) \hat{\sigma}_{-}(t)\right\rangle+2 \sqrt{\lambda_{x}} w_{\tau}^{(x)}\left\langle\hat{\sigma}_{z}(t+\tau) \hat{\sigma}_{-}(t)\right\rangle \\
\frac{d}{d \tau}\left\langle\hat{\sigma}_{x}(t+\tau) \hat{\sigma}_{-}(t)\right\rangle=-\beta_{\mathrm{QED}}\left\langle\hat{\sigma}_{x}(t+\tau) \hat{\sigma}_{-}(t)\right\rangle-\left(\Omega_{\mathrm{QED}}-2 \sqrt{\lambda_{z}} w_{t}^{(z)}\right)\left\langle\hat{\sigma}_{y}(t+\tau) \hat{\sigma}_{-}(t)\right\rangle .
\end{gathered}
$$

Using the aforementioned theorems to switch between Stratonovich and Itô forms and also to obtain the stochastic expectations, we find

$$
\begin{gathered}
\frac{d}{d \tau} \mathbb{E}\left(\left\langle\hat{\sigma}_{z}(t+\tau) \hat{\sigma}_{-}(t)\right\rangle\right)=-2\left(\beta_{\mathrm{QED}}+\lambda_{x}\right) \mathbb{E}\left(\left\langle\hat{\sigma}_{z}(t+\tau) \hat{\sigma}_{-}(t)\right\rangle\right), \\
\frac{d}{d \tau} \mathbb{E}\left(\left\langle\hat{\sigma}_{y}(t+\tau) \hat{\sigma}_{-}(t)\right\rangle\right)=\Omega_{\mathrm{QED}} \mathbb{E}\left(\left\langle\hat{\sigma}_{x}(t+\tau) \hat{\sigma}_{-}(t)\right\rangle\right)-\left(\beta_{\mathrm{QED}}+2 \lambda_{x}+2 \lambda_{z}\right) \mathbb{E}\left(\left\langle\hat{\sigma}_{y}(t+\tau) \hat{\sigma}_{-}(t)\right\rangle\right), \\
\frac{d}{d \tau} \mathbb{E}\left(\left\langle\hat{\sigma}_{x}(t+\tau) \hat{\sigma}_{-}(t)\right\rangle\right)=-\left(\beta_{\mathrm{QED}}+2 \lambda_{z}\right) \mathbb{E}\left(\left\langle\hat{\sigma}_{x}(t+\tau) \hat{\sigma}_{-}(t)\right\rangle\right)-\Omega_{\mathrm{QED}} \mathbb{E}\left(\left\langle\hat{\sigma}_{y}(t+\tau) \hat{\sigma}_{-}(t)\right\rangle\right) .
\end{gathered}
$$

Accordingly, for the dipole-dipole autocorrelation function we get

$$
\begin{aligned}
\mathbb{E}\left(\left\langle\hat{\sigma}_{+}(t+\tau) \hat{\sigma}_{-}(t)\right\rangle\right) & =\frac{1}{2}\left[\mathbb{E}\left(\left\langle\hat{\sigma}_{x}(t+\tau) \hat{\sigma}_{-}(t)\right\rangle\right)+i \mathbb{E}\left(\left\langle\hat{\sigma}_{y}(t+\tau) \hat{\sigma}_{-}(t)\right\rangle\right)\right] \\
& =e^{-\left(\beta_{\mathrm{QED}}+\lambda_{x}+2 \lambda_{z}\right) \tau}\left(\cos \Omega \tau+i \frac{\Omega_{\mathrm{QED}}}{\Omega} \sin \Omega \tau\right) \mathbb{E}\left(\left\langle\hat{\sigma}_{+}(t) \hat{\sigma}_{-}(t)\right\rangle\right)
\end{aligned}
$$




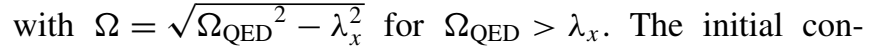
ditions that we used are $\mathbb{E}\left(\left\langle\hat{\sigma}_{x}(t) \hat{\sigma}_{-}(t)\right\rangle\right)=\mathbb{E}\left(\left\langle\hat{\sigma}_{+}(t) \hat{\sigma}_{-}(t)\right\rangle\right)$ and $\mathbb{E}\left(\left\langle\hat{\sigma}_{y}(t) \hat{\sigma}_{-}(t)\right\rangle\right)=-i \mathbb{E}\left(\left\langle\hat{\sigma}_{+}(t) \hat{\sigma}_{-}(t)\right\rangle\right)$. For $\Omega_{\mathrm{QED}} \gg \lambda_{x}$, which is the case for most cases of experimental interest, we can expand $\Omega_{\mathrm{QED}} / \Omega$ to the first leading term in $\lambda_{x} / \Omega_{\mathrm{QED}}$, and we get

$$
\begin{aligned}
\mathbb{E}\left(\left\langle\hat{\sigma}_{+}(t+\tau) \hat{\sigma}_{-}(t)\right\rangle\right) \simeq & e^{-\left(\beta_{\mathrm{QED}}+\lambda_{x}+2 \lambda_{z}\right) \tau} e^{i \Omega \tau} \\
& \times \mathbb{E}\left(\left\langle\hat{\sigma}_{+}(t) \hat{\sigma}_{-}(t)\right\rangle\right) .
\end{aligned}
$$

Using the Wiener-Khinchin relation, for the spectral density of emitted radiation we obtain

$$
\begin{aligned}
\mathcal{S}(\omega) & =\frac{1}{\pi}\left[\frac{\beta_{\mathrm{QED}}+\lambda_{x}+2 \lambda_{z}}{\left(\beta_{\mathrm{QED}}+\lambda_{x}+2 \lambda_{z}\right)^{2}+(\omega-\Omega)^{2}}\right] \\
& \equiv \frac{1}{\pi}\left[\frac{\beta}{\beta^{2}+(\omega-\Omega)^{2}}\right],
\end{aligned}
$$

with the full width at half maximum of $\beta$ where

$$
\beta=\beta_{\mathrm{QED}}+\beta_{\mathrm{N}}, \quad \text { with } \quad \beta_{\mathrm{N}}=\lambda_{x}+2 \lambda_{z},
$$

and the central frequency of

$$
\Omega=\sqrt{\Omega_{\mathrm{QED}}^{2}-\lambda_{x}^{2}} \simeq \Omega_{\mathrm{QED}}-\gamma_{\mathrm{N}}, \quad \text { with } \quad \gamma_{\mathrm{N}}=\frac{\lambda_{x}^{2}}{2 \omega_{0}} .
$$

Accordingly, the radiative corrections given by violations of the quantum superposition principle produce two observable effects: a frequency shift and a line broadening, whose magnitude is controlled by the rates $\lambda_{x, z}$. Their numerical value depends on the specific model used to describe nonlinear (collapse) effects.

Accordingly, a violation of the usual quantum dynamics manifests both as frequency shift and broadening. Measuring such quantities with sufficient accuracy, besides being interesting per se, amounts also to a test of the quantum superposition principle. Note that, since decoherence is also usually described by the Lindblad Eq. (2), external perturbations also induce shift and broadening in the spectrum [51]. Therefore, like in matter-wave or optomechanical interferometry, a system must be suitably screened from surrounding noises.

To detect small deviations from standard quantum predictions, one needs ultrahigh-resolution spectroscopic techniques, with a quality factor $(Q=\omega / \beta)$ of the spectroscopic system as high as possible. The state of the art for the frequency stability of a laser $(1 / Q)$ is of the order $10^{-16}$ in the visible spectral range [52], which can be transferred to other frequency domains, in principle, by frequency combs [53]. However, most recently the frequency stability for an atomic frequency measurement in the visible-near-infrared spectral domain has been measured to resolve instabilities even on the the order of $10^{-18}$ [54]. Such technical capabilities are very promising to observe spectral effects.

\section{QUANTIFYING THE SHIFT AND THE BROADENING FOR THE CSL AND DP MODELS}

We now derive the collapse broadening $\beta_{\mathrm{N}}$ and shift $\gamma_{\mathrm{N}}$ as predicted by the two most-studied collapse models in the literature: the mass proportional CSL model [13] and the DP model [11].

\section{A. Rates for the CSL model}

The stochastic potential $\hat{V}_{t}$ associated to the CSL model is [13]

$$
\hat{V}_{t}=-\frac{\hbar \sqrt{\gamma}}{m_{0}} \int d \mathbf{x} \xi_{t}(\mathbf{x}) \hat{L}(\mathbf{x})
$$

with

$$
\hat{L}(\mathbf{x})=\int d \mathbf{y} g(\mathbf{x}-\mathbf{y}) \sum_{\mathfrak{j}} m_{\mathfrak{j}} \sum_{s} \hat{a}_{\mathfrak{j}}^{\dagger}(s, \mathbf{y}) \hat{a}_{\mathfrak{j}}(s, \mathbf{y}),
$$

where $m_{0}=1 \mathrm{amu}, \gamma \simeq 10^{-22} \mathrm{~cm}^{3} \mathrm{~s}^{-1}$ [55], $\xi_{t}(\mathbf{x})$ is a white noise with correlation $\mathbb{E}\left(\xi_{t}(\mathbf{x}) \xi_{\tau}(\mathbf{y})\right)=\delta(t-\tau) \delta(\mathbf{x}-\mathbf{y})$, and $\hat{a}_{\mathfrak{j}}(s, \mathbf{y})$ is the annihilation operator of the particle type $\mathfrak{j}$ with mass $m_{\mathfrak{j}}$ and the spin $s$ at position $\mathbf{y}$; and $g(\mathbf{r})=$ $\exp \left(-\mathbf{r}^{2} / 2 r_{C}^{2}\right) /\left(\sqrt{2 \pi} r_{C}\right)^{3}$, with $r_{C} \simeq 10^{-5} \mathrm{~cm}$ the correlation length.

In the two-level representation, the matrix elements of $\hat{V}_{t}$ are given by

$$
\begin{aligned}
V_{t}^{\alpha \beta}=\left\langle\varepsilon_{\alpha}\left|\hat{V}_{t}\right| \varepsilon_{\beta}\right\rangle= & -\frac{\hbar \sqrt{\gamma}}{m_{0}} \int d \mathbf{Q} \psi_{\alpha}(\mathbf{Q}) \psi_{\beta}(\mathbf{Q}) \\
& \times \int d \mathbf{x} \xi_{t}(\mathbf{x}) \sum_{j=1}^{N} m_{j} g\left(\mathbf{x}-\mathbf{q}_{j}\right),
\end{aligned}
$$

with $\alpha, \beta=1,2$ and $\psi_{\alpha}(\mathbf{Q})=\left\langle\mathbf{Q} \mid \varepsilon_{\alpha}\right\rangle$, where we use improper states, $|\mathbf{Q}\rangle \equiv\left|\mathbf{q}_{1} ; \mathbf{q}_{2} ; \ldots ; \mathbf{q}_{N}\right\rangle$ (with $\mathbf{q}_{j}$ the position of $j$ th particle), for which we have $\hat{L}(\mathbf{x})|\mathbf{Q}\rangle=\left[\sum_{j=1}^{N} m_{j} g\left(\mathbf{x}-\mathbf{q}_{j}\right)\right]|\mathbf{Q}\rangle$. We also assume that the wave functions $\psi_{\alpha}$ are real. Since the right side of Eq. (34) contains a Gaussian white noise, $\lambda_{x, z}$ can be calculated as follows:

$$
\begin{aligned}
\mathbb{E}\left(V_{t_{1}}^{\alpha \beta} V_{t_{2}}^{\alpha^{\prime} \beta^{\prime}}\right)= & \frac{\delta\left(t_{1}-t_{2}\right) \hbar^{2} \gamma}{8 \pi^{3 / 2} r_{C}^{3}} \\
& \times \int d \mathbf{Q} d \mathbf{Q}^{\prime} \psi_{\alpha}(\mathbf{Q}) \psi_{\beta}(\mathbf{Q}) \psi_{\alpha^{\prime}}\left(\mathbf{Q}^{\prime}\right) \psi_{\beta^{\prime}}\left(\mathbf{Q}^{\prime}\right) \\
& \times \sum_{j, l=1}^{N} \frac{m_{j} m_{l}}{m_{0}^{2}} \exp \left[-\frac{\left(\mathbf{q}_{j}-\mathbf{q}_{l}^{\prime}\right)^{2}}{4 r_{C}^{2}}\right] \\
= & \delta\left(t_{1}-t_{2}\right) \hbar^{2} \lambda_{\alpha^{\prime} \beta^{\prime}}^{\alpha \beta}
\end{aligned}
$$

We consider the situation where the effective size of the region in which $\psi_{1,2}$ is different from zero is smaller than $r_{C} \simeq 10^{-7}$ $\mathrm{m}$, which is the case for atomic and molecules systems. This is the small scale limit of the CSL model. Accordingly, by expanding the exponential term in Eq. (35) to first order in $\left(\mathbf{q}_{j}-\mathbf{q}_{l}^{\prime}\right)^{2} / 4 r_{C}^{2}$ and then by performing the integrations, we get

$$
\begin{aligned}
& \lambda_{11}^{11} \simeq \Upsilon\left[1-\int d \mathbf{Q}\left|\psi_{1}(\mathbf{Q})\right|^{2} \sum_{j} \frac{m_{j}}{M}\left(\frac{\mathbf{q}_{j}}{2 r_{C}}\right)^{2}\right], \\
& \lambda_{22}^{22} \simeq \Upsilon\left[1-\int d \mathbf{Q}\left|\psi_{2}(\mathbf{Q})\right|^{2} \sum_{j} \frac{m_{j}}{M}\left(\frac{\mathbf{q}_{j}}{2 r_{C}}\right)^{2}\right], \\
& \lambda_{21}^{21}=\lambda_{12}^{12} \simeq \Upsilon\left[\int d \mathbf{Q} \psi_{1}(\mathbf{Q}) \psi_{2}(\mathbf{Q}) \sum_{j} m_{j} \mathbf{q}_{j}\right]^{2},
\end{aligned}
$$


with $\Upsilon=\Lambda_{\mathrm{CSL}}\left(M / m_{0}\right)^{2}, \quad \Lambda_{\mathrm{CSL}}=\gamma /\left(8 \pi^{3 / 2} r_{C}^{3}\right)=1.12 \times$ $10^{-9} \mathrm{~s}^{-1}$, and $M=\sum_{j} m_{j}$. In the derivation of the above equations, we used the parity considerations and the orthogonality of $\psi_{1}$ and $\psi_{2}$. Accordingly, we have

$$
\begin{aligned}
\lambda_{z}=\frac{\lambda_{11}^{11}-\lambda_{22}^{22}}{2}= & \frac{\Lambda_{\mathrm{CSL}} M}{8 r_{C}^{2} m_{0}^{2}} \\
& \times \int d \mathbf{Q}\left[\left|\psi_{2}(\mathbf{Q})\right|^{2}-\left|\psi_{1}(\mathbf{Q})\right|^{2}\right] \sum_{j} m_{j} \mathbf{q}_{j}^{2},
\end{aligned}
$$$$
\lambda_{x}=\lambda_{12}^{12}=\frac{\Lambda_{\mathrm{CSL}}}{2 r_{C}^{2} m_{0}^{2}}\left[\int d \mathbf{Q} \psi_{1}(\mathbf{Q}) \psi_{2}(\mathbf{Q}) \sum_{j} m_{j} \mathbf{q}_{j}\right]^{2} .
$$

\section{B. Rates for the Diósi-Penrose model}

The stochastic potential in the DP model is given by

$$
\hat{V}_{t}=-\hbar \int d \mathbf{x} \xi_{t}(\mathbf{x}) \hat{L}(\mathbf{x})
$$

where $\hat{L}(\mathbf{x})$ is the same like the CSL model, and $\xi_{t}(\mathbf{x})$ is a white noise with correlation $\mathbb{E}\left(\xi_{t}(\mathbf{x}) \xi_{s}(\mathbf{y})\right)=G \delta(t-s) / \hbar|\mathbf{x}-\mathbf{y}|$, with $G$ the gravitational constant. This form of $\hat{L}(\mathbf{x})$ is different from Diósi's original proposal [11]. To avoid the divergence due to the Newton self-energy, Diósi initially proposed a Lindblad operator with a length cutoff equal to the nuclear size. Then, it was shown [56] that with this cutoff, predictions of the DP model are in contradiction with known observations. To avoid this problem, Ghirardi, Grassi, and Rimini [56] proposed this new form of Lindblad operator whose cutoff is $r_{C} \simeq 10^{-7} \mathrm{~m}$. This adjustment of the model was eventually acknowledged by Diósi [57].

For the matrix elements of $\hat{V}_{t}$ in two-level representation, one gets

$$
\begin{aligned}
V_{t}^{\alpha \beta}=\left\langle\varepsilon_{\alpha}\left|\hat{V}_{t}\right| \varepsilon_{\beta}\right\rangle= & \hbar \int d \mathbf{Q} \psi_{\alpha}(\mathbf{Q}) \psi_{\beta}(\mathbf{Q}) \\
& \times \int d \mathbf{x} \xi_{t}(\mathbf{x}) \sum_{j=1}^{N} m_{j} g\left(\mathbf{x}-\mathbf{q}_{j}\right) .
\end{aligned}
$$

Following the same approach that we used for the CSL model, we find

$$
\begin{aligned}
& \mathbb{E}\left(V_{t_{1}}^{\alpha \beta} V_{t_{2}}^{\alpha^{\prime} \beta^{\prime}}\right)= \delta\left(t_{1}-t_{2}\right) G \hbar \int d \mathbf{Q} d \mathbf{Q}^{\prime} \psi_{\alpha}(\mathbf{Q}) \psi_{\beta}(\mathbf{Q}) \psi_{\alpha^{\prime}} \\
& \times\left(\mathbf{Q}^{\prime}\right) \psi_{\beta^{\prime}}\left(\mathbf{Q}^{\prime}\right) \sum_{j, l} m_{j} m_{l} \\
& \times \int \frac{d \mathbf{x} d \mathbf{x}^{\prime}}{\left|\mathbf{x}-\mathbf{x}^{\prime}\right|} g\left(\mathbf{x}-\mathbf{q}_{j}\right) g\left(\mathbf{x}^{\prime}-\mathbf{q}_{l}^{\prime}\right) \\
&=\frac{\delta\left(t_{1}-t_{2}\right) G \hbar}{4 \pi} \int d \mathbf{Q} d \mathbf{Q}^{\prime} \psi_{\alpha}(\mathbf{Q}) \psi_{\beta}(\mathbf{Q}) \psi_{\alpha^{\prime}}\left(\mathbf{Q}^{\prime}\right) \psi_{\beta^{\prime}}\left(\mathbf{Q}^{\prime}\right) \\
& \quad \times \sum_{j, l} m_{j} m_{l} \int d \mathbf{x} g(\mathbf{x}) \Phi_{j l}(\mathbf{x})=\delta\left(t_{1}-t_{2}\right) \hbar^{2} \lambda_{\alpha^{\prime} \beta^{\prime}}^{\alpha \beta},
\end{aligned}
$$

with

$$
\Phi_{j l}(\mathbf{x})=\frac{\operatorname{erf}\left[\frac{\mid \mathbf{x}-\left(\mathbf{q}_{j}-\mathbf{q}_{l}^{\prime}\right)}{\sqrt{2} r_{C}}\right]}{\left|\mathbf{x}-\left(\mathbf{q}_{j}-\mathbf{q}_{l}^{\prime}\right)\right|},
$$

where erf is the error function. Here $\Phi_{j l}(\mathbf{x})$ is slowly varying with respect to $g(\mathbf{x})$. Therefore, $g(\mathbf{x})$ acts like a Dirac $\delta$, practically selecting the value of $\Phi_{j l}(\mathbf{x})$ in the origin $x=0$. Accordingly, we can write

$$
\begin{aligned}
\mathbb{E}\left(V_{t_{1}}^{\alpha \beta} V_{t_{2}}^{\alpha^{\prime} \beta^{\prime}}\right) \simeq & \frac{\delta\left(t_{1}-t_{2}\right) G \hbar}{4 \pi} \int d \mathbf{Q} d \mathbf{Q}^{\prime} \psi_{\alpha}(\mathbf{Q}) \psi_{\beta}(\mathbf{Q}) \psi_{\alpha^{\prime}} \\
& \times\left(\mathbf{Q}^{\prime}\right) \psi_{\beta^{\prime}}\left(\mathbf{Q}^{\prime}\right) \sum_{j, l} m_{j} m_{l} \frac{\operatorname{erf}\left(\frac{\left|\mathbf{q}_{j}-\mathbf{q}_{l}^{\prime}\right|}{\sqrt{2} r_{c}}\right)}{\left|\mathbf{q}_{j}-\mathbf{q}_{l}^{\prime}\right|} .
\end{aligned}
$$

Like before, we are interested in the cases where the spatial width of eigenenergies $\psi_{1,2}$ are smaller than $r_{C}$, meaning $\mid \mathbf{q}_{j}-$ $\mathbf{q}_{l}^{\prime} \mid \ll r_{C}$. This implies that $\Phi_{j l}(\mathbf{0})$ can be Taylor expanded, and to the leading order, one finds

$$
\frac{\operatorname{erf}\left(\frac{\left|\mathbf{q}_{j}-\mathbf{q}_{l}^{\prime}\right|}{\sqrt{2} r_{C}}\right)}{\left|\mathbf{q}_{j}-\mathbf{q}_{l}^{\prime}\right|} \simeq \frac{2}{r_{C} \sqrt{2 \pi}}\left(1-\frac{\left|\mathbf{q}_{j}-\mathbf{q}_{l}^{\prime}\right|^{2}}{6 r_{C}^{2}}\right),
$$

where $\left|\mathbf{q}_{j}-\mathbf{q}_{l}^{\prime}\right| \ll r_{C}$. Then one can follow the same line of reasoning that we followed from Eq. (35) to Eqs. (39) and (40) to solve the rest of integrations. Accordingly, the rates $\lambda_{x, z}$ of the DP model become

$$
\begin{gathered}
\lambda_{z}=\frac{\Lambda_{\mathrm{DP}} M}{8 r_{C}^{2} m_{0}^{2}} \int d \mathbf{Q}\left[\left|\psi_{2}(\mathbf{Q})\right|^{2}-\left|\psi_{1}(\mathbf{Q})\right|^{2}\right] \sum_{j} m_{j} \mathbf{q}_{j}^{2}, \\
\lambda_{x}=\lambda_{12}^{12}=\frac{\Lambda_{\mathrm{DP}}}{2 r_{C}^{2} m_{0}^{2}}\left[\int d \mathbf{Q} \psi_{1}(\mathbf{Q}) \psi_{2}(\mathbf{Q}) \sum_{j} m_{j} \mathbf{q}_{j}\right]^{2},
\end{gathered}
$$

with $\Lambda_{\mathrm{DP}}=\frac{G m_{0}^{2}}{3 \sqrt{2} \pi^{3 / 2} \hbar r_{C}}$. Using the above results, for the noise shift and broadening we finally get

$$
\begin{gathered}
\beta_{\mathrm{N}}=\frac{\Lambda}{2 r_{C}^{2} m_{0}^{2}}\left[D_{12}^{2}+\frac{M}{2}\left(I_{2}-I_{1}\right)\right], \\
\gamma_{\mathrm{N}}=\frac{\Lambda^{2}}{8 \omega_{0}}\left(\frac{D_{12}}{r_{C} m_{0}}\right)^{4},
\end{gathered}
$$

with $I_{\alpha}=\left\langle\varepsilon_{\alpha}\left|\left(\sum_{j=1}^{N} m_{j} \hat{\mathbf{q}}_{j}^{2}\right)\right| \varepsilon_{\alpha}\right\rangle$ the average momentum of inertia ( $\alpha=1,2$ and $N$ the total number of particles), $\mathbf{D}_{12}=$ $\left\langle\varepsilon_{2}\left|\left(\sum_{j=1}^{N} m_{j} \hat{\mathbf{q}}_{j}\right)\right| \varepsilon_{1}\right\rangle, M=\sum_{j=1}^{N} m_{j}$ the total mass, and $\Lambda$ the coupling constant with the collapse or gravitational field. For the CSL model we get $\Lambda_{\mathrm{CSL}} \simeq 1.12 \times 10^{-9} \mathrm{~s}^{-1}$ and for the DP model $\Lambda_{\mathrm{DP}} \simeq 7.39 \times 10^{-25} \mathrm{~Hz}$. From now on, we consider only CSL, as the effect of DP model is by far smaller.

\section{APPLICATION TO RELEVANT PHYSICAL SYSTEMS}

We now provide quantitative estimates of these rates for some interesting physical systems.

Hydrogenlike atoms. For an atom that contains only one electron, we have $\mathbf{D}_{12}=\left(m_{e} / e\right) \mathbf{d}_{12}$, with $m_{e}$ the mass, $e$ the 
charge of electron, and $\mathbf{d}_{12}$ the off-diagonal element of the dipole moment with typical values of a few debye. In addition, $\sqrt{I_{\alpha} / m_{e}}$ has typical values of a few Bohr radius. Accordingly, we get

$$
\beta_{\mathrm{N}} \sim 10^{-20}-10^{-18} \mathrm{~s}^{-1} ; \quad \gamma_{\mathrm{N}} \sim 10^{-53} \mathrm{~s}^{-1} .
$$

Harmonic oscillator. We consider the two lowest states of a harmonic oscillator with mass $\mu$ and frequency $\omega_{0}$. Introducing these eigenstates into Eqs. (39) and (40) and performing the integration, we find

$$
\beta_{\mathrm{N}}=3 \sqrt{2 \omega_{0} \gamma_{\mathrm{N}}}=\frac{3 \Lambda}{4}\left(\frac{\mu x_{0}}{m_{0} r_{C}}\right)^{2},
$$

with $x_{0}=\sqrt{\hbar / \mu \omega_{0}}$ the zero-point fluctuation amplitude.

Double-well potential. We consider a system of mass $\mu$ moving in a symmetric double-well potential at low temperatures, where the meaningful eigenstates are the two lowest ones: $\left|\varepsilon_{1}\right\rangle=\frac{1}{\sqrt{2}}(|R\rangle+|L\rangle)$ and $\left|\varepsilon_{2}\right\rangle=\frac{1}{\sqrt{2}}(|R\rangle-|L\rangle)$. The tunneling frequency is $\omega_{0}=\left(\varepsilon_{2}-\varepsilon_{1}\right) / \hbar$. We denote the separation of minima by $q_{0}$. The states $|L\rangle$ and $|R\rangle$ are localized states at left and right minima, respectively. They can be transformed to each other by the displacement operator where the displacement distance is $q_{0}$, and $\langle L \mid R\rangle \simeq 0$. Accordingly, using Eqs. (39) and (40) and performing the integration, we find

$$
\beta_{\mathrm{N}}=\sqrt{2 \omega_{0} \gamma_{\mathrm{N}}}=\frac{\Lambda}{8}\left(\frac{\mu q_{0}}{m_{0} r_{C}}\right)^{2} .
$$

The estimated values of collapse broadening shift systems for these system are reported in Table I.

\section{ESTIMATING DECOHERENCE EFFECTS}

In typical experiments, there are spectral broadening effects due to external noises, which dominate the shape and width of spectral lines. The effects reduce the lifetime $\tau$ of the coherently excited state, but can be maintained by controlling limiting environmental parameters as temperature and pres- sure, or by experimental arrangements. We give numbers for collisional and Doppler broadening for a vibrational mode of a generic system (mass of system $M=10^{7} \mathrm{amu}, \omega_{0}=10^{13} \mathrm{~Hz}$ at $T=10 \mathrm{~K}$ ).

Collisional broadening happens when emission is triggered by collision with other particles. A simple expression for the effective collision rate is $\beta_{\mathrm{C}} \sim n \sigma_{\mathrm{C}} v_{\text {th }}$, with $n$ the density of a bath, $\sigma_{\mathrm{C}}$ the effective cross section of colliding particles, and $v_{\text {th }}$ the thermal relative velocity. For $n=$ $10^{12}$ particles $/ \mathrm{m}^{3}$ (laboratory vacuum) and air molecules as a bath, we find $\beta_{\mathrm{C}} \sim \mathrm{mHz}$ in the hard-sphere limit. For more complicated interaction potential (such as London dispersion), we have showed that the decoherence rate is of order $\beta_{\mathrm{C}} \sim 10^{-4}-10^{-2} \mathrm{~Hz}$ for a double-well potential system at typical vacuum conditions as achieved in today's experiments [58].

Doppler broadening is an effect of the thermal motion of an ensemble of emitters and is given by [45] $\beta_{\mathrm{D}}=\omega_{0} \sqrt{\frac{2 k_{\mathrm{B}} T \ln 2}{M c^{2}}} \simeq$ $3568 \mathrm{~Hz}$. However, saturation spectroscopy can be used to avoid Doppler broadening. Therefore, both decoherence effects can be distinguished from the desired collapse effect. We use this here as a qualitative argument to argue how to separate collapse broadening from systematic effects in a given experimental realization of the test.

\section{A. Universality of broadening}

A crucial feature of Eqs. (50) and (51) is that both broadening and shift induced by collapse models are universal in the sense that they depend only on the mass of the system (at the practical level, only on the mass of those particles, whose position changes significantly during the transition) and on the geometry of the levels and nothing else. This has to be compared with decoherence broadening and shift, which depends also on the details of the surrounding environment: mass of the bath particle, cross section, pressure, temperature. Moreover, in the case of collapse models, they roughly scale quadratically with the mass of the system, while the mass dependence with decoherence is different.

TABLE I. Collapse broadening and shift as predicted by the CSL model. Three relevant situations have been considered: the transition from the $2 p$ to $1 s$ state in a hydrogenlike atom; the transition from the first excited state to the ground state for a harmonic potential; and a double-well potential (see Secs. II-V for a description of these systems). The latter case is particularly relevant to describe chiral molecules. The constant $\Lambda \simeq 1.12 \times 10^{-9} \mathrm{~Hz}$ measures the strength of the collapse (see main text), $m_{0}=1 \mathrm{amu}, r_{C} \simeq 10^{-7} \mathrm{~m}$ is the correlation length of the noise inducing the collapse, $x_{0}=\sqrt{\hbar / \mu \omega_{0}}$ is the zero-point width of harmonic oscillator, and $q_{0}$ is the separation of the minima of double-well potential. For the double-well potential, we assume the range of the molecular vibration: $\omega_{0} \sim 10^{12}-10^{14} \mathrm{~Hz}$. We have considered only the predictions of the CSL model, since those of the DP model are much smaller. All numbers in this table are exemplary to illustrate the magnitude of the spectral effects and not necessarily realized in experiments yet.

\begin{tabular}{lcc}
\hline \hline System & $\beta_{\mathrm{N}}(\mathrm{Hz})$ & $\gamma_{\mathrm{N}}(\mathrm{Hz})$ \\
\hline Hydrogenlike atoms & $10^{-20}-10^{-18}$ & $\sim 10^{-53}$ \\
Harmonic oscillator & $\frac{3 \Lambda}{4}\left(\frac{\mu x_{0}}{m_{0} r_{C}}\right)^{2}$ & $\frac{\Lambda^{2}}{32 \omega_{0}}\left(\frac{\mu x_{0}}{m_{0} r_{C}}\right)^{4}$ \\
$\mu=1$ amu and $\omega_{0}=10^{10} \mathrm{~Hz}$ & $5.3 \times 10^{-13}$ & $6.2 \times 10^{-36}$ \\
$\mu=10^{7}$ amu and $\omega_{0}=1.7 \times 10^{8} \mathrm{~Hz}$ & $3.1 \times 10^{-4}$ & $1.3 \times 10^{-16}$ \\
Double well & $\frac{\Lambda}{8}\left(\frac{\mu q_{0}}{m_{0} r_{C}}\right)^{2}$ & $\Lambda^{2}$ \\
$\mu=m_{e}=5.5 \times 10^{-4}$ amu and $q_{0}=1 \AA$ & 4 \\
$m_{0} r_{C}$ & 128 \\
$\mu=1$ amu and $q_{0}=1 \AA$ & $4.2 \times 10^{-23}$ & $10^{-57}-10^{-55}$ \\
$\mu=10^{7}$ amu and $q_{0}=1 \AA$ & $1.4 \times 10^{-16}$ & $10^{-44}-10^{-42}$ \\
\hline
\end{tabular}


(a)

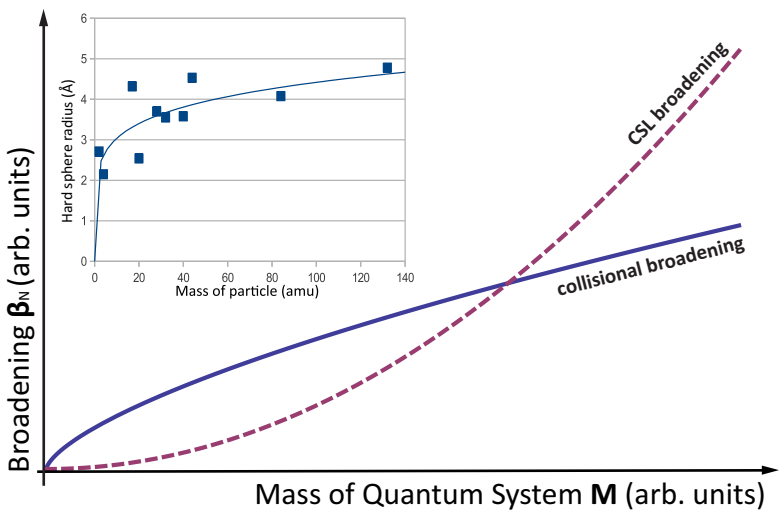

(b)

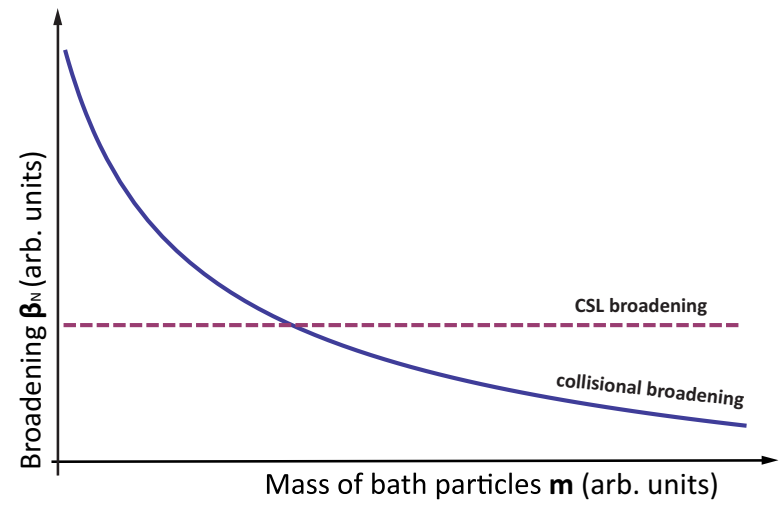

FIG. 2. (Color online) Collapse models predicts that the broadening $\beta_{\mathrm{N}}$ depends only on the geometry of involved states and scales quadratically with the mass of the system [see Eq. (50)]. On the other hand, decoherence broadening, in general, scales differently with the mass and moreover depends on the details of the interaction with the environment. In panel (a) we show the different scaling behavior between CSL broadening and decoherence broadening (in the recoil-free regime $M \gg m$ ), while the environment does not change and the system increases in mass. Assuming that the density of the system remains constant so that $r \propto M^{1 / 3}$ ( $r$ is the radius of the systems and $M$ its mass), then in the hard-sphere limit one has for collisional broadening [45]: $\beta_{\mathrm{C}}=4 d^{2} p \sqrt{\pi / m k_{\mathrm{B}} T} \propto M^{2 / 3}$ (where $d \sim r$ is the closet distance among the colliding particles, $p$ the pressure of the bath, $T$ its temperature, and $m$ the mass of bath particles). In the inset, the dependence of the experimental hard-sphere radius on mass is shown, for $\mathrm{H}_{2}, \mathrm{He}, \mathrm{NH}_{3}, \mathrm{Ne}, \mathrm{N}_{2}, \mathrm{O}_{2}, \mathrm{Ar}, \mathrm{CO}_{2}, \mathrm{Kr}$, and Xe [59]. The behavior is qualitatively similar to the oversimplified $M^{1 / 3}$ dependence we estimated; therefore, the mass dependence of $\beta_{\mathrm{C}}$ is different from the $M^{2}$ behavior of the CSL broadening. In panel (b) we assume the opposite situation. The system does not change and the bath particle increases in mass (still in the recoil-free regime $M \gg m$ ). In such a case, CSL broadening remains constant, while decoherence broadening scales as $m^{-1 / 2}$.

This behavior represents a specific signature, which can be used to discriminate collapse broadening from decoherence broadening (see Fig. 2).

\section{CONCLUSION}

Spectral effects originating from possible violations of the quantum superposition principle are small, but within reach of experimental observation. They are universal, which means omnipresent and not controllable by modifications of environmental influences on the two-level system, and can, in principle, be seen for the emission from any system, which can be approximated as a two-level system. This universal nature of the predicted effect is in contrast to spectral broadening and shift effects due to decoherence effects, which can be controlled by control of the interaction of the two-level system with the environment. However, to observe the here proposed spectral effects, macroscopicity parameters (mass, spatial size, and time of superposition) have to be chosen to allow for a relevant magnitude of the effect. The standard QED analysis of photon emission was perturbed by a collapse noise term to include the effect of nonlinearity into the equations.

Thermal and collisional decoherence effects can be reduced to the required limitations and distinguished according to their scaling with experimental parameters. We expect that this holds true for any other systematic effect in experiments sensitive enough to probe the predicted effect. While we cannot predict the ultimate two-level system here, we give explicit examples to illustrate the relation to state-of-the-art experiments.

Today, ultrahigh-resolution spectroscopy in the midinfrared spectral range is done with a precision of $10^{-13}$ [60] and is planned to be improved to $10^{-16}$ [61]. Spectral CSL effects are of the order of $10^{-14}$ to $10^{-16}$ for a double-well system of $10^{7}$ amu and $q_{0}=1 \AA$ (see Table I) probed in the midinfrared range. This test seems feasible in the near future. Further electron or nuclear spins are known to be accessible with ultrahigh-frequency resolution of $10 \mathrm{mHz}$. The Larmor frequency of the system ${ }^{7} \mathrm{Li}^{+}$free induction decay (FID) in water, would need to be detected with a spectral resolution of about $30 \mu \mathrm{Hz}$ to show a CSL effect, which is only three orders of magnitude away from today's resolution [62]. Further relevant are solid-state systems, like semiconductor microcavities, quantum dots, or nanodiamonds with vacancy centers and spin structure [63] as well as optomechanical systems.

Therefore, we speculate that experimental realization is well within reach, if the interest of experts can be attracted to the here-described general effect, which should, in principe, be visible in any two-level system in the defined parameter regime. To be able to measure a spectral line broadening according to nonlinear modifications of the Schrödinger equation will have quite significant impact on the foundations of physics, as it would prove the validity of the assumption that a universal decoherence mechanism exists and is the reason for the observed quantum-to-classical transition of macroscopic systems. This then-solidified fact would allow for further speculations of the nature of a new theory underlying the quantum one.

\section{ACKNOWLEDGMENTS}

M.B. and A.B. acknowledge financial support from EU Rroject NANOQUESTFIT. A.B. wishes to thank the COST Action No. MP1006 "Fundamental Problems in Quantum Physics." A.B. acknowledges partial support from INFN. 
M.B. thanks Professor M. Ghasem Mahjani of K. N. Toosi University, Iran, for his valuable supports. H.U. thanks the U.K. funding agency EPSRC (Grant No. EP/J014664/1), the Foundational Questions Institute (FQXi), and the John
F. Templeton Foundation for financial support. All authors thank Dr. B. Darquié of LPL, Université Paris 13, France, for his valuable comments on experimental part of this paper.
[1] E. Joos, H. D. Zeh, C. Kiefer, D. J. W. Giulini, J. Kupsch, and I.-O. Stamatescu, Decoherence and the Appearance of a Classical World in Quantum Theory, 2nd ed. (Springer, Berlin, 2003).

[2] H.-P. Breuer and F. Petruccione, The Theory of Open Quantum Systems (Oxford University Press, Oxford, UK, 2002).

[3] K. Hornberger, S. Uttenthaler, B. Brezger, L. Hackermüller, M. Arndt, and A. Zeilinger, Phys. Rev. Lett. 90, 160401 (2003).

[4] L. Hackermller, K. Hornberger, B. Brezger, A. Zeilinger, and M. Arndt, Nature (London) 427, 711 (2004).

[5] S. Nimmrichter and K. Hornberger, Phys. Rev. Lett. 110, 160403 (2013).

[6] S. Eibenberger, S. Gerlich, M. Arndt, M. Mayor, and J. Tüxen, Phys. Chem. Chem. Phys. 15, 14696 (2013).

[7] A. J. Leggett, J. Phys.: Condens. Matter 14, R415 (2002).

[8] S. L. Adler and A. Bassi, Science 325, 275 (2009).

[9] S. L. Adler, Quantum Theory as an Emergent Phenomenon (Cambridge University Press, Cambridge, UK, 2004).

[10] S. Weinberg, Phys. Rev. A 85, 062116 (2012).

[11] L. Diósi, Phys. Lett. A 120, 377 (1987); R. Penrose, Gen. Rel. Grav. 28, 581 (1996).

[12] D. Giulini and A. Groardt, Class. Quantum Grav. 28, 195026 (2011); H. Yang, H. Miao, D. S. Lee, B. Helou, and Y. Chen, Phys. Rev. Lett. 110, 170401 (2013).

[13] G. C. Ghirardi, A. Rimini, and T. Weber, Phys. Rev. D 34, 470 (1986); G. C. Ghirardi, P. Pearle, and A. Rimini, Phys. Rev. A 42, 78 (1990); G. C. Ghirardi, R. Grassi, and F. Benatti, Found. Phys. 25, 5 (1995).

[14] A. Bassi and G. C. Ghirardi, Phys. Rep. 379, 257 (2003).

[15] A. Bassi, K. Lochan, S. Satin, T. P. Singh, and H. Ulbricht, Rev. Mod. Phys. 85, 471 (2013).

[16] S. Nimmrichter, K. Hornberger, P. Haslinger, and M. Arndt, Phys. Rev. A 83, 043621 (2011).

[17] K. Hornberger, S. Gerlich, P. Haslinger, S. Nimmrichter, and M. Arndt, Rev. Mod. Phys. 84, 157 (2012).

[18] J. R. Friedman, M. P. Sarachik, J. Tejada, and R. Ziolo, Phys. Rev. Lett. 76, 3830 (1996).

[19] W. Wernsdorfer, E. Bonet Orozco, K. Hasselbach, A. Benoit, D. Mailly, O. Kubo, H. Nakano, and B. Barbara, Phys. Rev. Lett. 79, 4014 (1997).

[20] Y. Nakamura, Yu. A. Pashkin, and J. S. Tsai, Nature (London) 398, 786 (1999).

[21] C. H. van der Wal et al., Science 290, 773 (2000).

[22] J. R. Friedman et al., Nature (London) 406, 43 (2000).

[23] S. Gerlich et al., Nat. Commun. 2, 263 (2011).

[24] P. Haslinger, N. Dörre, P. Geyer, J. Rodewald, S. Nimmrichter, and M. Arndt, Nat. Phys. 9, 144 (2013).

[25] T. Juffmann, H. Ulbricht, and M. Arndt, Rep. Prog. Phys. 76, 086402 (2013).

[26] W. Marshall, C. Simon, R. Penrose, and D. Bouwmeester, Phys. Rev. Lett. 91, 130401 (2003).
[27] O. Romero-Isart, A. C. Pflanzer, F. Blaser, R. Kaltenbaek, N. Kiesel, M. Aspelmeyer, and J. I. Cirac, Phys. Rev. Lett. 107, 020405 (2011).

[28] S. Nimmrichter, P. Haslinger, K. Hornberger, and M. Arndt, New J. Phys. 13, 075002 (2011).

[29] J. Bateman, S. Nimmrichter, K. Hornberger, and H. Ulbricht, arXiv:1312.0500v1.

[30] N. Kiesel et al., Proc. Natl. Acad. Sci. USA 110, 14180 (2013).

[31] P. Asenbaum, S. Kuhn, S. Nimmrichter, U. Sezer, and M. Arndt, Nat. Commun. 4, 2743 (2013).

[32] A. Xuereb, H. Ulbricht, and M. Paternostro, Sci. Rep. 3, 3378 (2013).

[33] M. Scala, M. S. Kim, G. W. Morley, P. F. Barker, and S. Bose, Phys. Rev. Lett. 111, 180403 (2013).

[34] Z-Q. Yin, T. Li, X. Zhang, and L. M. Duan, Phys. Rev. A 88, 033614 (2013).

[35] R. Kaltenbaek et al., Exp. Astron. 34, 123 (2012).

[36] N. Gisin, Hel. Phys. Acta 62, 363 (1989); Phys. Lett. A 143, 1 (1990).

[37] N. Gisin and M. Rigo, J. Phys. A: Math. Gen. 28, 7375 (1995).

[38] J. Polchinski, Phys. Rev. Lett. 66, 397 (1991).

[39] A. Bassi, D. Durr, and G. Hinrichs, Phys. Rev. Lett. 111, 210401 (2013)

[40] N. Gisin, Phys. Rev. Lett. 52, 1657 (1984).

[41] L. Diósi, Phys. Lett. A 129, 419 (1988).

[42] S. L. Adler and L. P. Horwitz, J. Math. Phys. 41, 2485 (2000).

[43] S. L. Adler, arXiv:hep-th/0206120v1.

[44] S. L. Adler, Phys. Rev. D 67, 025007 (2003), added note.

[45] W. Heitler, Quantum Theory of Radiation, 3rd ed., International Series of Monographs on Physics (Clarendon, Oxford, UK, 1954), Secs. 16, 17, 18, and 34.

[46] G. S. Agarwal, Quantum Statistical Theories of Spontaneous Emission and their Relation to other Approaches, Springer Tracts in Modern Physics Vol. 70 (Springer, Berlin, 1974).

[47] J. C. Garrison and R. Y. Chiao, Quantum Optics (Oxford University Press, Oxford, UK, 2008), Chaps. 4 and 11.

[48] L. Mandel and E. Wolf, Optical Coherence and Quantum Optics (Cambridge University Press, Cambridge, UK, 1995), Chap. 15.

[49] H. A. Bethe, Phys. Rev. 72, 339 (1947).

[50] L. Arnold, Stochastic Differential Equations: Theory and Applications (Dover, New York, 2013).

[51] J. Hartmann, C. Boulet, and D. Robert, Collisional Effects on Molecular Spectra (Elsevier, Amsterdam, 2008).

[52] G. D. Cole et al., Nat. Photon. 7, 644 (2013).

[53] T. Udem, R. Holzwarth, and Th. W. Hänsch, Nature (London) 416, 233 (2002).

[54] N. Hinkley et al., Science 341, 1215 (2013).

[55] S. L. Adler, J. Phys. A: Math. Theor. 40, 2935 (2007). 
[56] G. C. Ghirardi, R. Grassi, and A. Rimini, Phys. Rev. A 42, 1057 (1990).

[57] L. Diósi, Braz. J. Phys. 35, 260 (2005); J. Phys. A: Math. Theor. 40, 2989 (2007).

[58] M. Bahrami, A. Shafiee, and A. Bassi, Phys. Chem. Chem. Phys. 14, 9214 (2012).

[59] D. R. Lide, ed., CRC Handbook of Chemistry and Physics, 77th ed. (CRC Press, Boca Raton, FL, 1996).
[60] C. Daussy, T. Marrel, A. Amy-Klein, C. T. Nguyen, C. J. Borde, and C. Chardonnet, Phys. Rev. Lett. 83, 1554 (1999).

[61] B. Darquí et al., Chirality 22, 870 (2010).

[62] S. Appelt, H. Kühn, F. W. Häsing, and B. Blümich, Nat. Phys. 2, 105 (2006).

[63] Y. Yamamoto and I. Imamoglu, Mesoscopic Quantum Optics (Wiley, New York, 1999). 